\title{
HLA-haploidentical stem cell transplantation using posttransplant cyclophosphamide
}

\author{
Junichi Sugita ${ }^{1}$ (D) \\ Received: 4 April 2019 / Revised: 9 May 2019 / Accepted: 9 May 2019 / Published online: 18 May 2019 \\ (c) Japanese Society of Hematology 2019
}

\begin{abstract}
HLA-haploidentical stem cell transplantation using posttransplant cyclophosphamide has spread rapidly worldwide. This strategy was initially developed in the setting of bone marrow transplantation following nonmyeloablative conditioning. Recently, peripheral blood stem cell grafts and/or myeloablative conditioning regimen have been widely used. In Japan, prospective, multicenter, phase II studies have been conducted by the Japan Study Group for Cell Therapy and Transplantation to evaluate the safety and efficacy of HLA-haploidentical peripheral blood stem cell transplantation using posttransplant cyclophosphamide (PTCy-haploPBSCT). In the first such study (JSCT Haplo 13 study), we demonstrated that PTCy-haploPBSCT after busulfan-based reduced-intensity conditioning (RIC) enables stable donor engraftment and low incidences of both acute and chronic graft-versus-host disease (GVHD). In the second (JSCT Haplo 14 study), we showed that both myeloablative conditioning (MAC) and RIC are valid options for PTCy-haploPBSCT. Emerging evidence, including our findings, suggests that donor type (HLA-haploidentical donor versus HLA-matched related or unrelated donor) may no longer be a significant predictor of transplant outcome.
\end{abstract}

Keywords HLA-haploidentical transplantation · Posttransplant cyclophosphamide

\section{Introduction}

Allogeneic hematopoietic stem cell transplantation (SCT) is a potentially curative treatment for patients with hematological malignancies and nonmalignant diseases. Historically, HLA matching has been the most important factor influencing the transplant outcome. Although SCT from HLA-matched donor leads to best outcome, HLA-matched related or unrelated donors are not always available. In contrast, HLA-haploidentical donors, who share a single HLA haplotype with recipients, are nearly always available. Therefore, HLA-haploidentical SCT (haploSCT) can be used for patients who lack HLA-matched donors and/or are in an urgent need of transplantation. Calcineurin inhibitor (CNI) and methotrexate (MTX) have been used as the

Junichi Sugita

sugitaj@med.hokudai.ac.jp

1 Department of Hematology, Faculty of Medicine, Hokkaido University, N15 W7, Kita-Ku, Sapporo 060-8638, Japan standard graft-versus-host disease (GVHD) prophylaxis in HLA-matched stem cell transplantation; however, haploSCT was originally associated with high incidences of GVHD and graft rejection [1-3]. Over the last few decades, several strategies have been developed to overcome HLA barriers in haploSCT.

T cells are the main mediators of GHVD, one of the most effective strategies to overcome HLA barriers in haploSCT is $\mathrm{T}$ cell depletion from the graft. The major approaches for $\mathrm{T}$-cell depletion are ex vivo $\mathrm{T}$-cell depletion using $\mathrm{CD} 34+$ positive selection and in vivo $\mathrm{T}$-cell depletion using antithymocyte globulin (ATG) $[4,5]$. However, these strategies are potentially associated with an increased risk of severe opportunistic infections and non-relapse mortality (NRM) early after SCT due to delayed immune reconstitution. More recently, T-cell replete haploSCT using posttransplant cyclophosphamide (PTCy-haploSCT) has been developed. Rationale of this strategy is assumed to be selective and cytotoxic depletion of alloreactive T cells, while preserving non-alloreactive $\mathrm{T}$ cells [6]. A series of previous studies by Johns Hopkins group have demonstrated that this 
strategy was feasible and safe with low incidences of acute and chronic GVHD and NRM [7, 8]. In this review, we summarize current clinical uses and issues of PTCy-haploSCT.

\section{Donor selection for PTCy-haploSCT}

Donor selection is an important issue because several haploidentical donors are available for most patients in the setting of haploSCT. With regard to the number of HLA mismatches, Lorentino et al. [9] demonstrated that no significant associations were found between cumulative number of HLA mismatches and severe acute GVHD, chronic GVHD, NRM, relapse rate, progression-free survival (PFS), or overall survival (OS) in patients who received haploSCT using PTCy or ATG. Raiola reported similar results in patients who received HLA-haploidentical bone marrow transplantation (PTCy-haploBMT). No correlations were observed between the number of HLA mismatches and OS, NRM, the incidence of acute GVHD, chronic GVHD, or graft rejection [10]. These results suggest that the degree of HLA mismatching is not essential to be counted for donor selection in PTCy-haploSCT. In other words, donor selection based on criteria other than HLA may improve transplant outcome. Younger donor age $(\leq 40$ years) may be a predictor for improved survival in older patients with AML and MDS receiving PTCy-haploSCT [11]. PTCy-haploBMT from non-first-degree relatives results is similar to those seen with first-degree relatives [12]. The impact of donor versus recipient killer cell immunoglobulin-like receptor (KIR)ligand mismatch in PTCy-haploSCT is still controversial [13-16].

One of the most important factors to select donor in haploSCT is donor-specific antibodies (DSA), which target mismatched donor HLAs. DSA have been recently recognized as an important factor for graft rejection in HLAmismatched SCT [17]. Therefore, all recipients should be screened for DSA before haploSCT, and the donor who has
HLA alleles targeted by DSA should be avoided. A positive test for DSA is considered when mean fluorescence intensity (MFI) is above 1000; however, the clinically relevant cutoff level remains to be unclear. MFI over 5000 was the relatively high risk for primary graft rejection in haploSCT [18]. In case the donor who has mismatched HLA targeted by DSA cannot be avoided, several strategies have developed for reduction of DSA, including plasmapheresis, intravenous gamma-globulin, rituximab, and bortezomib [18-20].

\section{Conditioning regimen and stem cell source for PTCy-haploSCT}

HLA-mismatch, myeloablative conditioning (MAC), and peripheral blood stem cell transplantation (PBSCT) are risk factors for GVHD [21]. Therefore, PTCy-haploSCT was initially developed in the setting of bone marrow transplantation (BMT) following nonmyeloablative conditioning (NMC) to reduce GVHD incidence [7, 8].

PTCy-haploSCT using MAC and reduced-intensity conditioning (RIC) has been developed to reduce relapse [22-28]. A variety of conditioning regimens are used in PTCy-haploSCT (Table 1). In the same way as HLAmatched SCT, MAC regimens contain total body irradiation (TBI) or busulfan (BU), RIC regimens contain fludarabine (Flu) and BU and/or melphalan (MEL).

Huselton et al. [29] reported the retrospective analysis comparing outcomes after haploSCT with MAC and RIC using propensity score. In this study, MAC regimens were associated with reduced relapse but increased NRM compared to RIC. There were no differences in OS, disease-free survival (DFS), or incidences of acute and chronic GVHD between MAC and RIC. These results indicate that both MAC and RIC are valid options for PTCy-haploSCT.

Because of the greater content of T cells in PBSC grafts compared with BM grafts, PBSCT may be associated with better disease control. Several groups have developed
Table 1 Conditioning regimens for HLA-haploidentical stem cell transplantation using posttransplant cyclophosphamide

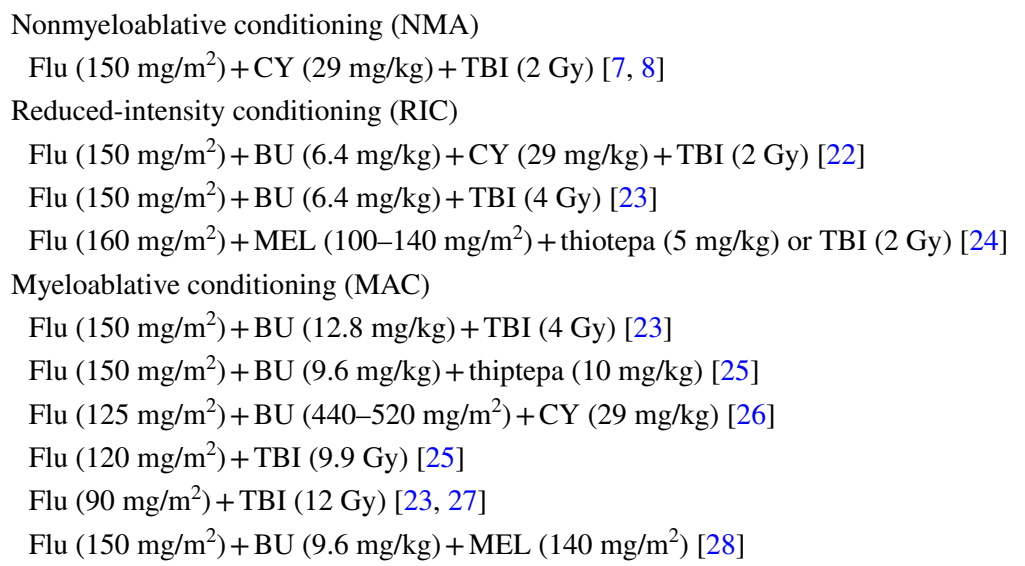


PTCy-haploPBSCT [22, 23, 25, 27, 28]. A recent retrospective comparison of PTCy-haploBMT and PTCy-haploPBSCT showed higher incidences of grade II-IV acute and chronic GVHD in patients who received PTCy-haploPBSCT [30]. Although OS was not affected by stem cell sources, patients who received PTCy-haploPBSCT had a lower incidence of relapse compared to those who received PTCy-haploBMT. Further studies are needed to assess the impact of stem cell sources on transplant outcome in PTCy-haploSCT.

\section{GVHD prophylaxis for PTCy-haploSCT}

The standard GVHD prophylaxis in PTCy-haploSCT consisted of cyclophosphamide (50 mg/kg/day on days 3 and 4) and tacrolimus plus mycophenolate mofetil (MMF) starting on day 5 [7, 8, 22-24, 26-28], several groups reported modified GVHD prophylaxes for PTCy-haploSCT (Table 2) [25, 31-34]. Castagna et al. [31] retrospectively analyzed patients who received tacrolimus and those who received cyclosporine. There were no major differences between tacrolimus and cyclosporine in PTCy-haploSCT. Bacigalupo et al. [25] modified the timing of cyclophosphamide administrated on days 3 and 5, and started cyclosporine and MMF before day 3. Although immunosuppressive drugs administrated before the infusion of PTCy may attenuate the effect of PTCy, possibly by inhibiting cell cycle and reducing sensitivities of donor alloreactive T cells to PTCY, it seems to be still effective to reduce the incidence of GVHD. As mentioned before, PTCy is combined with TAC + MMF in PTCy-haploSCT; however, PTCy could be used in combination with Tac alone, sirolimus + MMF, or TAC + MTX [32-34]. There was no prospective study comparing various GVHD prophylaxes that are to be combined with PTCY in PTCy-haploSCT. Further studies are needed to determine the best GVHD prophylaxis in PTCy-haploSCT.

\section{PTCY-haploSCT in Japan}

Several prospective, multicenter, phase II studies have been conducted by the Japan Study Group for Cell Therapy and
Transplantation (JSCT) to evaluate the safety and efficacy of PTCy-haploPBSCT in Japan [22, 23].

In the first study (JSCT Haplo 13 study), we added BU $(6.4 \mathrm{mg} / \mathrm{kg}$ ) to the original nonmyeloablative platform consisting of Flu, cyclophosphamide, and 2 Gy of TBI developed by Johns Hopkins group [7, 8, 22]. In addition, peripheral blood stem cell grafts were used instead of bone marrow grafts. Thirty-one patients with a median age of 48 (21-65) were enrolled in this study. Thirteen patients $(42 \%)$ had a history of prior allo-SCT. Neutrophil engraftment was achieved in $87 \%$ patients with a median of 19 days. The cumulative incidences of grades II-IV and III-IV acute GVHD and chronic GVHD at 1 year were $23 \%, 3 \%$, and $15 \%$, respectively. NRM was $19 \%$ at day 100 . The patients without a history of prior allo-SCT had higher engraftment (100\%) and lower NRM (11\%). This study suggests that PTCy-haploPBSCT after BU-containing RIC enables stable donor engraftment and low incidences of both acute and chronic GVHD.

As the second study (JSCT Haplo 14 study), we conducted two parallel prospective phase II studies of MACand RIC-based PTCy-haploPBSCT [23]. MAC regimen was either Flu ( $\left.90 \mathrm{mg} / \mathrm{m}^{2}\right)$ plus TBI (12 Gy), or a combination of Flu $\left(150 \mathrm{mg} / \mathrm{m}^{2}\right), \mathrm{BU}(12.8 \mathrm{mg} / \mathrm{kg})$, and $4 \mathrm{~Gy}$ of TBI (Fig. 1). RIC regimen was a combination of Flu $(150 \mathrm{mg} /$ $\mathrm{m}^{2}$ ), BU (6.4 mg/kg), and 4 Gy of TBI (Fig. 2). Fifty-three patients were enrolled in the MAC study, and 81 patients were enrolled in the RIC study. Neutrophil engraftment was achieved in $98 \%$ and $94 \%$ in the MAC and RIC groups, respectively. The incidences of grades II-IV and III-IV acute GVHD were $18 \%$ and $8 \%$ in the MAC group, and $14 \%$ and $5 \%$ in the RIC group, respectively. Incidences of all grade and moderate to severe chronic GVHD at 2 years were $36 \%$ and $20 \%$ in the MAC group, and $27 \%$ and $20 \%$ in the RIC group, respectively. NRM at 2 years was $10 \%$ in the MAC group, and $20 \%$ in the RIC group. Notably, $83 \%$ and $86 \%$ of patients who survived without relapse successfully weaned off immunosuppressants by 2 years in the MAC and RIC groups, respectively. This study indicates that both MAC and RIC are valid options for PTCy-haploPBSCT.
Table 2 GVHD prophylaxes for HLA-haploidentical stem cell transplantation using posttransplant cyclophosphamide

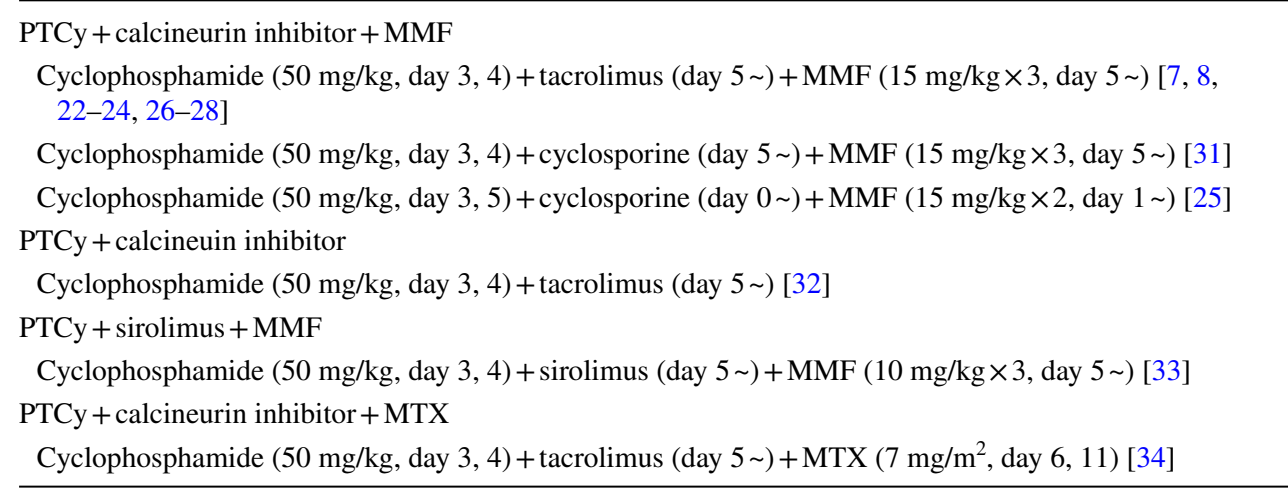


Fig. 1 Conditioning regimens and GVHD prophylaxis for JSCT Haplo 14 MAC study. JSCT Haplo 14 MAC regimen was either fludarabine (Flu $90 \mathrm{mg} / \mathrm{m}^{2}$ ) plus total body irradiation (TBI $12 \mathrm{~Gy}$ ), or a combination of Flu $(150 \mathrm{mg} /$ $\mathrm{m}^{2}$ ), busulfan (BU $12.8 \mathrm{mg} / \mathrm{kg}$ ), and TBI (4 Gy). Transplantation was performed on day 0 with unmanipulated peripheral blood stem cells. GVHD prophylaxis consisted of cyclophosphamide (CY; $50 \mathrm{mg} / \mathrm{kg} /$ day on days 3 and 4) and tacrolimus plus mycophenolate mofetil (MMF) starting on day 5

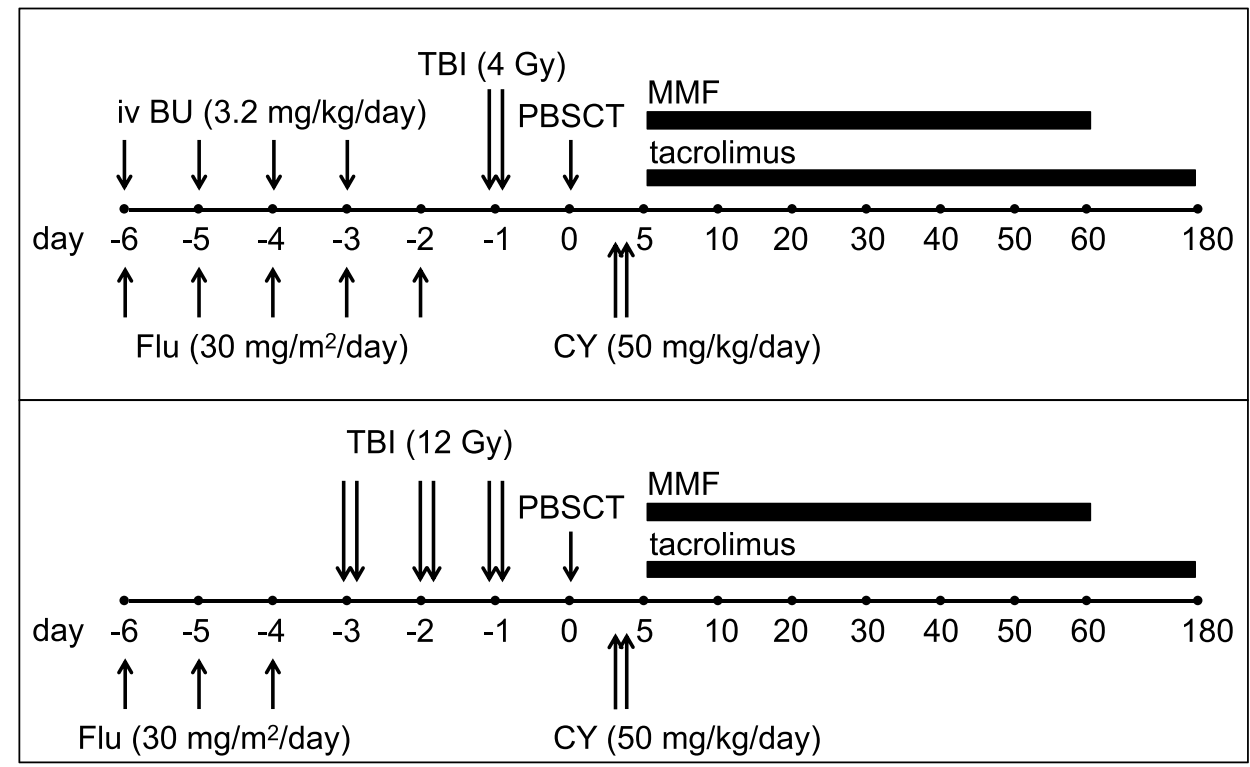

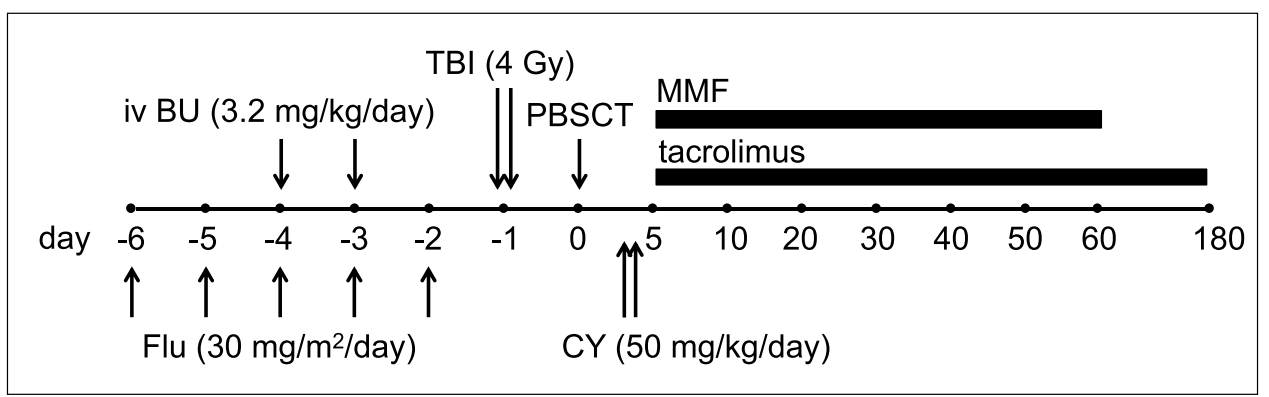

Fig. 2 Conditioning regimen and GVHD prophylaxis for JSCT Haplo 14 RIC study. JSCT Haplo 14 RIC regimen was a combination of fludarabine (Flu $150 \mathrm{mg} / \mathrm{m}^{2}$ ), busulfan (BU $6.4 \mathrm{mg} / \mathrm{kg}$ ), and otal body irradiation (TBI 4 Gy). Transplantation was performed on day 0 with

In addition to JSCT studies, there are several studies of PTCy-haploSCT in Japan [35, 36]. Nakamae et al. reduced the dose of PTCy to $25 \mathrm{mg} / \mathrm{kg}$ on day 3; however, they escalated the dose of PTCy to $25 \mathrm{mg} / \mathrm{kg}$ on days 3 and 4 because of a high incidence of grades III-IV acute GVHD. PTCy-haploPBSCT with $25 \mathrm{mg} / \mathrm{kg}$ of PTCy might be associated with a higher incidence of severe acute GVHD. It remains to be elucidated whether dose reduction of PTCy could be feasible. Recently, Nakamae and Fukuda et al. have conducted a multicenter, phase II study of PTCy-haploPBSCT using MEL-based RIC regimen for patients with adult T-cell leukemia/lymphoma (UMIN000021783). In this study, GVHD prophylaxis consisted of cyclophosphamide $(50 \mathrm{mg} / \mathrm{kg} /$ day on days 3 and 5$)$, tacrolimus (starting on day 1), and MMF (starting on day 6). To the best of our knowledge, this is the first study to evaluate the safety and efficacy of PTCy-haploPBSCT for patients with adult T-cell leukemia/lymphoma. unmanipulated peripheral blood stem cells. GVHD prophylaxis consisted of cyclophosphamide (CY; $50 \mathrm{mg} / \mathrm{kg} /$ day on days 3 and 4 ) and tacrolimus plus mycophenolate mofetil (MMF) starting on day 5

\section{Cytokine release syndrome after PTCy-haploSCT}

One of the major complications after PTCy-haploSCT is cytokine release syndrome (CRS). CRS is considered to be caused by rapid activation and proliferation of alloreactive donor $\mathrm{T}$ cells and the elevation of several cytokines, including IL-6, TNF- $\alpha$, and IFN- $\gamma$ [37]. The incidence of CRS differs by stem cell source. Almost all patients developed CRS with a peak at day 3 after MAC- and RIC-based PTCy-haploPBSCT [22, 23]. On the other hand, McCurdy et al. [38] reported that CRS occurred in 44\% after NMA-based PTCyhaploBMT, and 84\% after MAC-based PTCy-haploBMT. Raj et al. [39] demonstrated that PBSCT was significantly associated with grade $\geq 2$ CRS, compared with BMT. With regard to the risk of CRS, McCurdy et al. reported that CRS after PTCy-haploBMT is associated with myeloablation, HLA-class II mismatching, and higher $\mathrm{CD} 3+$ graft cell dose. Although CRS is mild (grades 1 or 2) in most patients and 
resolved quickly after administration of PTCy, some patients may experience severe (grades 3 or 4 ) CRS. McCurdy et al. reported that $12 \%$ of patients developed severe CRS and they had worse survival [40]. Serum IL-6 levels were elevated in CRS; anti-IL-6 receptor therapy (tocilizumab) can be beneficial for resolution of the CRS symptoms. It is still unknown whether this phenomenon has antitumor effect.

\section{Cardiotoxicity after PTCy-haploSCT}

High-dose cyclophosphamide can cause acute cardiotoxicity characterized by hemorrhagic necrotic perimyocarditis [41]. The incidence was $<1-9 \%$ after the most commonly used high-dose cyclophosphamide $(60 \mathrm{mg} / \mathrm{kg}$ daily $\times 2$ days or $50 \mathrm{mg} / \mathrm{kg}$ daily $\times 4$ days). Although cardiotoxicity was historically reported mainly after high-dose cyclophosphamide as conditioning regimen, there is little known about the cardiotoxicity after PTCy $(50 \mathrm{mg} / \mathrm{kg}$ daily $\times 2$ days $)$. It remains unclear whether cardiomyopathy is more common in patients after PTCy-haploSCT.

\section{Comparison with HLA-haploidentical stem cell transplantation using ATG}

Several studies demonstrated better outcomes with PTCyhaploSCT compared to ATG-based haploSCT. Ruggeri et al. compared the use of PTCy and ATG as GVHD prophylaxis for haploSCT in patients with acute myeloid leukemia (AML) in complete remission. This study demonstrated that patients receiving PTCy had better leukemia-free survival (LFS) and GVHD-free, relapse-free survival (GRFS), lower incidence of GVHD, and lower NRM than those receiving ATG [42]. Li et al. [43] also demonstrated that patients receiving ATG resulted in higher NRM and inferior LFS, OS, and GRFS in patients with secondary acute myeloid leukemia compared to those receiving PTCy. The incidences of relapse were not significantly different in these studies.

\section{Comparison with umbilical cord blood transplantation}

When compared with umbilical cord blood transplantation (CBT), PTCy-haploSCT demonstrated equal or even better results either in retrospective or parallel prospective studies [43-47].

Ruggeri et al. [44] retrospectively compared the outcomes after single or double CBT and PTCy-haploSCT in adults with de novo AML and acute lymphoblastic leukemia (ALL). Although CBT was associated with delayed engraftment and higher graft failure, there were no statistical differences on main outcomes including relapse rate, NRM, and LFS. Gauthier et al. [45] reported that PTCy-haploSCT in patients with Hodgkin lymphoma was associated with a better GRFS compared to mismatched unrelated or single or double CBT, mainly due to the reduced incidence of grade III-IV acute GVHD and chronic GVHD.

The Blood and Marrow Transplant Clinical Trials Network (BMT CTN) conducted two parallel multicenter phase 2 trials to evaluate the use of double CBT and PTCy-haploBMT after RIC (BMT CTN 0603, BMT CTN 0604) [46, 47]. The incidence of grade II-IV acute GVHD was $40 \%$ after CBT and $32 \%$ after PTCy-haploBMT. OS, PFS, NRM, and relapse rate at 3 years were $39 \%, 36 \%, 28 \%$, and $36 \%$ after CBT, and $54 \%, 35 \%, 8 \%, 58 \%$ after PTCy-haploBMT. These results confirmed the safety and effectiveness of these alternative allograft sources in the multicenter setting. A large multicenter phase III randomized trial comparing double unit CBT versus PTCy-haploBMT is currently ongoing (BMT CTN 1101; NCT0159778).

PTCy-haploSCT might be beneficial in patients with graft rejection after CBT. Singh et al. [48] reported 21 consecutive patients who experienced graft failure after CBT. Twelve patients received a second SCT, six from separate CBT and six from a haploidentical donor. Of the six long-term survivors without relapse, four received a second HSCT from a haploidentical donor with PTCy.

\section{Comparison with HLA-matched SCT}

Several retrospective studies using registry data from Center for International Blood and Marrow Transplant Research (CIBMTR) or European Society for Blood and Marrow Transplantation (EBMT) indicated that OS and PFS after PTCyhaploSCT were comparable with transplantation from HLAmatched related donor (MRD) or HLA-matched unrelated donor (MUD) in patients with acute leukemia and lymphoma (Table 3) [49-53]. Notably, almost all of these studies demonstrated the lower incidence of chronic GVHD in patients after PTCy-haploSCT along with similar relapse rates.

Meta-analysis by $\mathrm{Gu}$ et al. [54] verified these results. Nine case-control studies included 2258 patients (827 cases in the haplo-HCT with PT-Cy group; 748 controls in the MRD group; and 683 controls in the MUD group). They compared outcomes of PTCy-haploSCT with those of HLA-matched SCT. No differences were found between PTCy-haploSCT and HLA-matched SCT with regard to incidences of acute GVHD, NRM, relapse rate, PFS, and OS; however, PTCyhaploSCT was associated with a significantly lower incidence of moderate to severe chronic GVHD.

\section{Conclusion}

PTCy-haploSCT has spread rapidly worldwide because HLA-haploidentical donors are nearly always available and PTCy is dramatically effective and cheap, not requiring 


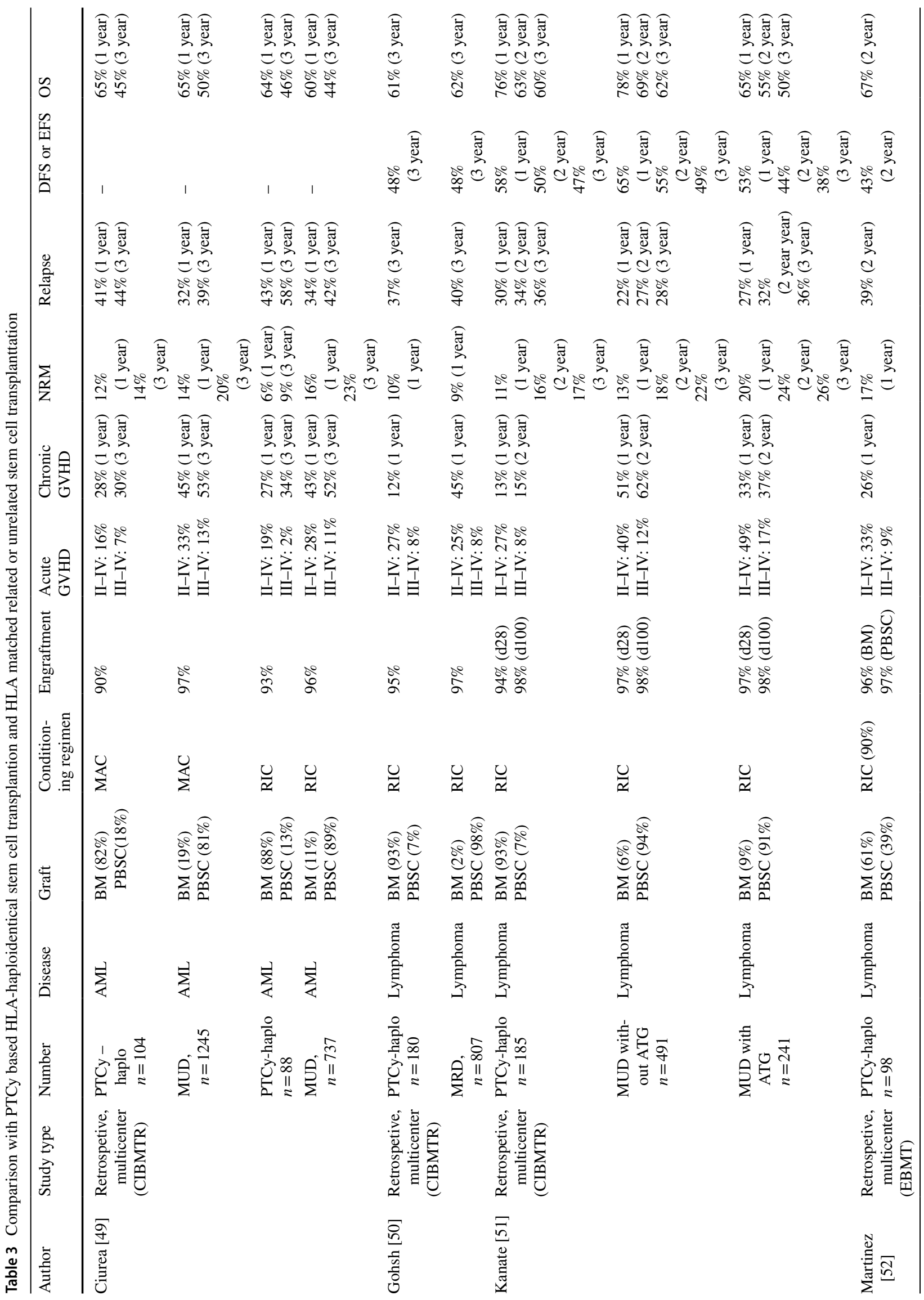


special expertise. Donor type (HLA-haploidentical donor vs. MRD or MUD) may no longer be a significant predictor of transplant outcome in the era of PTCy. Furthermore, recent studies demonstrated that this PTCy strategy could be applied in HLA-matched SCT. In Japan, PTCy is only used in the setting of clinical study, because high-dose cyclophosphamide is only approved as conditioning regimen. Prompt approval is needed for the use of PTCy as GVHD prophylaxis in Japan.

Acknowledgements This work was supported by the grant from Regional Medicine Research Foundation (Tochigi, Japan), North Japan Hematology Study Group (NJHSG), and Japan Agency for Medical Research and Development (AMED, JP17ek0510012).

\section{Compliance with ethical standards}

Conflict of interest The author declares no conflict of interest.

\section{References}

1. Beatty PG, Clift RA, Mickelson EM, Nisperos BB, Flournoy N, Martin PJ, et al. Marrow transplantation from related donors other than HLA-identical siblings. N Engl J Med. 1985;313:765-71.

2. Powles RL, Morgenstern GR, Kay HE, McElwain TJ, Clink HM, Dady PJ, et al. Mismatched family donors for bone-marrow transplantation as treatment for acute leukaemia. Lancet. 1983;1:612-5.

3. Anasetti C, Amos D, Beatty PG, Appelbaum FR, Bensinger W, Buckner CD, et al. Effect of HLA compatibility on engraftment of bone marrow transplants in patients with leukemia or lymphoma. N Engl J Med. 1989;320:197-204.

4. Aversa F, Tabilio A, Velardi A, Cunningham I, Terenzi A, Falzetti F, et al. Treatment of high-risk acute leukemia with T-celldepleted stem cells from related donors with one fully mismatched HLA haplotype. N Engl J Med. 1998;339:1186-93.

5. Lu D-P, Dong L, Wu T, Huang X-J, Zhang M-J, Han W, et al. Conditioning including antithymocyte globulin followed by unmanipulated HLA-mismatched/haploidentical blood and marrow transplantation can achieve comparable outcomes with HLAidentical sibling transplantation. Blood. 2006;107:3065-73.

6. Kanakry CG, Fuchs EJ, Luznik L. Modern approaches to HLAhaploidentical blood or marrow transplantation. Nat Rev Clin Oncol. 2016;13:10-24.

7. Luznik L, O'Donnell PV, Symons HJ, Chen AR, Leffell MS, Zahurak M, et al. HLA-haploidentical bone marrow transplantation for hematologic malignancies using nonmyeloablative conditioning and high-dose, posttransplantation cyclophosphamide. Biol Blood Marrow Transpl. 2008;14:641-50.

8. McCurdy SR, Kanakry JA, Showel MM, Tsai H-L, BolañosMeade J, Rosner GL, et al. Risk-stratified outcomes of nonmyeloablative HLA-haploidentical BMT with high-dose posttransplantation cyclophosphamide. Blood. 2015;125:3024-31.

9. Lorentino F, Labopin M, Fleischhauer K, Ciceri F, Mueller CR, Ruggeri A, et al. The impact of HLA matching on outcomes of unmanipulated haploidentical HSCT is modulated by GVHD prophylaxis. Blood Adv. 2017;1:669-80.

10. Raiola AM, Risitano A, Sacchi N, Giannoni L, Signori A, Aquino $\mathrm{S}$, et al. Impact of HLA disparity in haploidentical bone marrow transplantation followed by high-dose cyclophosphamide. Biol Blood Marrow Transpl. 2018;24:119-26. 
11. Ciurea SO, Shah MV, Saliba RM, Gaballa S, Kongtim P, Rondon $\mathrm{G}$, et al. Haploidentical transplantation for older patients with acute myeloid leukemia and myelodysplastic syndrome. Biol Blood Marrow Transpl. 2018;24:1232-6.

12. Elmariah H, Kasamon YL, Zahurak M, Macfarlane KW, Tucker $\mathrm{N}$, Rosner GL, et al. Haploidentical bone marrow transplantation with post-transplant cyclophosphamide using non-first-degree related donors. Biol Blood Marrow Transpl. 2018;24:1099-102.

13. Shimoni A, Labopin M, Lorentino F, et al. Killer cell immunoglobulin-like receptor ligand mismatching and outcome after haploidentical transplantation with post-transplant cyclophosphamide. Leukemia. 2019;33:230-9.

14. Torío A, Pascual MJ, Vidales I, Ortiz M, Caballero A, Heiniger AI. Donor selection based on killer cell immunoglobulin-like receptor (KIR) genotype may improve outcome after T-cell-replete haploidentical transplantation. Transpl Proc. 2018;50:679-82.

15. Wanquet A, Bramanti S, Harbi S, Fürst S, Legrand F, Faucher C, et al. Killer cell immunoglobulin-like receptor-ligand mismatch in donor versus recipient direction provides better graft-versus-tumor effect in patients with hematologic malignancies undergoing allogeneic T cell-replete haploidentical transplantation followed by post-transplant cyclophosphamide. Biol Blood Marrow Transpl. 2018;24:549-54.

16. Solomon SR, Aubrey MT, Zhang X, Piluso A, Freed BM, Brown $\mathrm{S}$, et al. Selecting the best donor for haploidentical transplant: impact of HLA, killer cell immunoglobulin-like receptor genotyping, and other clinical variables. Biol Blood Marrow Transpl. 2018;24:789-98.

17. Ciurea SO, Cao K, Fernandez-Vina M, Kongtim P, Malki MA, Fuchs E, et al. The European Society for Blood and Marrow Transplantation (EBMT) consensus guidelines for the detection and treatment of donor-specific anti-HLA antibodies (DSA) in haploidentical hematopoietic cell transplantation. Bone Marrow Transpl. 2018;53:521-34.

18. Ciurea SO, Thall PF, Milton DR, Barnes TH, Kongtim P, Carmazzi Y, et al. Complement-binding donor-specific anti-HLA antibodies and risk of primary graft failure in hematopoietic stem cell transplantation. Biol Blood Marrow Transpl. 2015;21:1392-8.

19. Kongtim P, Cao K, Ciurea SO. Donor specific anti-HLA antibody and risk of graft failure in haploidentical stem cell transplantation. Adv Hematol. 2016;2016:4025073-10.

20. Shiratori S, Ito M, Yoneoka M, Hayasaka K, Hayase E, Iwasaki J, et al. Successful engraftment in HLA-mismatched bone marrow transplantation despite the persistence of high-level donor-specific anti-HLA-DR antibody. Transplantation. 2013;96:e34-44.

21. Jagasia M, Arora M, Flowers MED, Chao NJ, McCarthy PL, Cutler CS, et al. Risk factors for acute GVHD and survival after hematopoietic cell transplantation. Blood. 2012;119:296-307.

22. Sugita J, Kawashima N, Fujisaki T, Kakihana K, Ota S, Matsuo $\mathrm{K}$, et al. HLA-haploidentical peripheral blood stem cell transplantation with post-transplant cyclophosphamide after busulfancontaining reduced-intensity conditioning. Biol Blood Marrow Transpl. 2015;21:1646-52.

23. Sugita J, Kagaya Y, Miyamoto T, et al. Myeloablative and reduced-intensity conditioning in HLA-haploidentical peripheral blood stem cell transplantation using post-transplant cyclophosphamide. Bone Marrow Transpl. 2019;54(3):432-41.

24. Brammer JE, Khouri I, Gaballa S, Anderlini P, Tomuleasa C, Ahmed S, et al. Outcomes of haploidentical stem cell transplantation for lymphoma with melphalan-based conditioning. Biol Blood Marrow Transpl. 2016;22:493-8.

25. Bacigalupo A, Dominietto A, Ghiso A, Di Grazia C, Lamparelli $\mathrm{T}$, Gualandi F, et al. Unmanipulated haploidentical bone marrow transplantation and post-transplant cyclophosphamide for hematologic malignancies following a myeloablative conditioning: an update. Bone Marrow Transpl. 2015;50:S37-9.
26. Solomon SR, Sizemore CA, Sanacore M, Zhang X, Brown S, Holland HK, et al. Haploidentical Transplantation using T cell replete peripheral blood stem cells and myeloablative conditioning in patients with high-risk hematologic malignancies who lack conventional donors is well tolerated and produces excellent relapse-free survival: results of a prospective phase II trial. Biol Blood Marrow Transpl. 2012;18:1859-66.

27. Solomon SR, Sizemore CA, Sanacore M, Zhang X, Brown S, Holland HK, et al. Total body irradiation-based myeloablative haploidentical stem cell transplantation is a safe and effective alternative to unrelated donor transplantation in patients without matched sibling donors. Biol Blood Marrow Transpl. 2015;21:1299-307.

28. Jaiswal SR, Chakrabarti A, Chatterjee S, Bhargava S, Ray K, Chakrabarti S. Hemophagocytic syndrome following haploidentical peripheral blood stem cell transplantation with post-transplant cyclophosphamide. Int J Hematol. 2016;103:234-42.

29. Huselton E, Slade M, Trinkaus KM, et al. Propensity score analysis of conditioning intensity in peripheral blood haploidentical hematopoietic cell transplantation. Biol Blood Marrow Transpl. 2018;24:2047-55.

30. Bashey A, Zhang M-J, McCurdy SR, St Martin A, Argall T, Anasetti C, et al. Mobilized peripheral blood stem cells versus unstimulated bone marrow as a graft source for T-cell-replete haploidentical donor transplantation using post-transplant cyclophosphamide. J Clin Oncol. 2017;35:3002-9.

31. Castagna L, Bramanti S, Fürst S, Giordano L, Sarina B, Crocchiolo R, et al. Tacrolimus compared with cyclosporine A after haploidentical T-cell replete transplantation with post-infusion cyclophosphamide. Bone Marrow Transpl. 2016;51:462-5.

32. Esquirol A, Pascual MJ, Ortiz M, et al. Single-agent GvHD prophylaxis with tacrolimus after post-transplant high-dose cyclophosphamide is a valid option for haploidentical transplantation in adults with hematological malignancies. Bone Marrow Transpl. 2017;52:1273-9.

33. Cieri N, Greco R, Crucitti L, Morelli M, Giglio F, Levati G, et al. Post-transplantation cyclophosphamide and sirolimus after haploidentical hematopoietic stem cell transplantation using a treosulfan-based myeloablative conditioning and peripheral blood stem cells. Biol Blood Marrow Transpl. 2015;21:1506-14.

34. Ohata K, Aoki G, Takamatsu H, Ishiyama K, Kondo Y, Yamazaki $\mathrm{H}$, et al. GVHD prophylaxis with short course MTX and tacrolimus in HLA-haploidentical hematopoietic stem cell transplantation using post-transplant high dose cyclophosphamide. Biol Blood Marrow Transpl. 2016;22:374.

35. Nakamae H, Koh H, Katayama T, et al. HLA haploidentical peripheral blood stem cell transplantation using reduced dose of posttransplantation cyclophosphamide for poor-prognosis or refractory leukemia and myelodysplastic syndrome. Exp Hematol. 2015;43:921-9.

36. Sawada A, Shimizu M, Isaka K, et al. Feasibility of HLA-haploidentical hematopoietic stem cell transplantation with post-transplantation cyclophosphamide for advanced pediatric malignancies. Pediatr Hematol Oncol. 2014;31:754-64.

37. Lee DW, Gardner R, Porter DL, Louis CU, Ahmed N, Jensen $\mathrm{M}$, et al. Current concepts in the diagnosis and management of cytokine release syndrome. Blood. 2014;124:188-95.

38. McCurdy SR, Muth ST, Tsai H-L, et al. Early fever after haploidentical bone marrow transplantation correlates with class II HLA-mismatching and myeloablation but not outcomes. Biol Blood Marrow Transpl. 2018;24:2056-64.

39. Raj RV, Hamadani M, Szabo A, et al. Peripheral blood grafts for T-cell replete haploidentical transplantation increase the incidence and severity of cytokine release syndrome. Biol Blood Marrow Transpl. 2018;24:1664-70.

40. Abboud R, Keller J, Slade M, DiPersio JF, Westervelt P, Rettig $\mathrm{MP}$, et al. Severe cytokine-release syndrome after T cell-replete 
peripheral blood haploidentical donor transplantation is associated with poor survival and anti-IL-6 therapy is safe and well tolerated. Biol Blood Marrow Transpl. 2016;22:1851-60.

41. Lin C-J, Vader JM, Slade M, DiPersio JF, Westervelt P, Romee R. Cardiomyopathy in patients after posttransplant cyclophosphamide-based hematopoietic cell transplantation. Cancer. 2017;123:1800-9.

42. Ruggeri A, Sun Y, Labopin M, Bacigalupo A, Lorentino F, Arcese W, et al. Post-transplant cyclophosphamide versus anti-thymocyte globulin as graft- versus-host disease prophylaxis in haploidentical transplant. Haematologica. 2017;102:401-10.

43. Li Z, Labopin M, Ciceri F, Blaise D, Tischer J, Ehninger G, et al. Haploidentical transplantation outcomes for secondary acute myeloid leukemia: acute Leukemia Working Party (ALWP) of the European Society for Blood and Marrow Transplantation (EBMT) study. Am J Hematol. 2018;93:769-77.

44. Ruggeri A, Labopin M, Sanz G, Piemontese S, Arcese W, Bacigalupo A, et al. Comparison of outcomes after unrelated cord blood and unmanipulated haploidentical stem cell transplantation in adults with acute leukemia. Leukemia. 2015;29:1891-900.

45. Gauthier J, Castagna L, Garnier F, Guillaume T, Socie G, Maury $\mathrm{S}$, et al. Reduced-intensity and non-myeloablative allogeneic stem cell transplantation from alternative HLA-mismatched donors for Hodgkin lymphoma: a study by the French Society of Bone Marrow Transplantation and Cellular Therapy. Bone Marrow Transpl. 2017;52:689-96.

46. Brunstein CG, Fuchs EJ, Carter SL, Karanes C, Costa LJ, Wu $\mathrm{J}$, et al. Alternative donor transplantation after reduced intensity conditioning: results of parallel phase 2 trials using partially HLAmismatched related bone marrow or unrelated double umbilical cord blood grafts. Blood. 2011;118:282-8.

47. Eapen M, O'Donnell P, Brunstein CG, Wu J, Barowski K, Mendizabal A, et al. Mismatched related and unrelated donors for allogeneic hematopoietic cell transplantation for adults with hematologic malignancies. Biol Blood Marrow Transpl. 2014;20:1485-92.

48. Singh H, Nikiforow S, Li S, et al. Outcomes and management strategies for graft failure after umbilical cord blood transplantation. Am J Hematol. 2014;89:1097-101.
49. Ciurea SO, Zhang M-J, Bacigalupo AA, Bashey A, Appelbaum FR, Aljitawi OS, et al. Haploidentical transplant with posttransplant cyclophosphamide vs matched unrelated donor transplant for acute myeloid leukemia. Blood. 2015;126:1033-40.

50. Ghosh N, Karmali R, Rocha V, Ahn KW, DiGilio A, Hari PN, et al. Reduced-intensity transplantation for lymphomas using haploidentical related donors versus HLA-matched sibling donors: a Center for International Blood and Marrow Transplant Research Analysis. J Clin Oncol. 2016;34:3141-9.

51. Kanate AS, Mussetti A, Kharfan-Dabaja MA, Ahn KW, DiGilio A, Beitinjaneh A, et al. Reduced-intensity transplantation for lymphomas using haploidentical related donors vs HLA-matched unrelated donors. Blood. 2016;127:938-47.

52. Martinez C, Gayoso J, Canals C, Finel H, Peggs K, Dominietto A, et al. Post-transplantation cyclophosphamide-based haploidentical transplantation as alternative to matched sibling or unrelated donor transplantation for Hodgkin lymphoma: a registry study of the Lymphoma Working Party of the European Society for Blood and Marrow Transplantation. J Clin Oncol. 2017;35:3425-32.

53. Brissot E, Labopin M, Ehninger G, et al. Haploidentical versus unrelated allogeneic stem cell transplantation for relapsed/refractory acute myeloid leukemia: a report on 1578 patients from the Acute Leukemia Working Party of the EBMT. Haematologica. 2019;104:524-32.

54. Gu Z, Wang L, Yuan L, et al. Similar outcomes after haploidentical transplantation with post-transplant cyclophosphamide versus HLA-matched transplantation: a meta-analysis of case-control studies. Oncotarget. 2017;8:63574-86.

Publisher's Note Springer Nature remains neutral with regard to jurisdictional claims in published maps and institutional affiliations. 